Original article

\title{
Primary care physician decision making regarding severe obesity treatment and bariatric surgery: A qualitative study
}

\author{
Luke M. Funk, M.D., M.P.H., ${ }^{\text {a, }}$, Sally A. Jolles, M.A. ${ }^{\text {a,b }}$, Caprice C. Greenberg, M.D., M.P.H. , \\ Margaret L. Schwarze, M.D. ${ }^{a}$, Nasia Safdar, M.D., Ph.D. ${ }^{\text {b,c }}$, Megan A. McVay, Ph.D. ${ }^{\text {d,e }}$, \\ Jeffrey C. Whittle, M.D., M.P.H. ${ }^{f}$, Matthew L. Maciejewski, Ph.D. ${ }^{e, g}$, Corrine I. Voils, Ph.D. ${ }^{e, g}$
}

${ }^{a}$ Department of Surgery, Wisconsin Surgical Outcomes Research Program (WiSOR), University of Wisconsin-Madison, Madison, Wisconsin

${ }^{b}$ William S. Middleton Veterans Memorial Hospital, Madison, Wisconsin

${ }^{c}$ Department of Medicine, University of Wisconsin-Madison, Madison, Wisconsin

${ }^{d}$ Department of Psychiatry and Behavioral Science, Duke University Medical Center, Durham, North Carolina

${ }^{e}$ Durham VA Medical Center, Health Services Research \& Development, Durham, North Carolina

${ }^{f}$ Clement J. Zablocki VA Medical Center, Milwaukee, Wisconsin

${ }^{g}$ Department of Medicine, Duke University Medical Center, Durham, North Carolina

Received July 22, 2011; accepted November 26, 2015

Abstract

Background: Less than $1 \%$ of severely obese US adults undergo bariatric surgery annually. It is critical to understand the factors that contribute to its utilization.

Objectives: To understand how primary care physicians (PCPs) make decisions regarding severe obesity treatment and bariatric surgery referral.

Setting: Focus groups with PCPs practicing in small, medium, and large cities in Wisconsin.

Methods: PCPs were asked to discuss prioritization of treatment for a severely obese patient with multiple co-morbidities and considerations regarding bariatric surgery referral. Focus group sessions were analyzed by using a directed approach to content analysis. A taxonomy of consensus codes was developed. Code summaries were created and representative quotes identified.

Results: Sixteen PCPs participated in 3 focus groups. Four treatment prioritization approaches were identified: (1) treat the disease that is easiest to address; (2) treat the disease that is perceived as the most dangerous; (3) let the patient set the agenda; and (4) address obesity first because it is the common denominator underlying other co-morbid conditions. Only the latter approach placed emphasis on obesity treatment. Five factors made PCPs hesitate to refer patients for bariatric surgery: (1) wanting to "do no harm"; (2) questioning the long-term effectiveness of bariatric surgery; (3) limited knowledge about bariatric surgery; (4) not wanting to recommend bariatric surgery too early; and (5) not knowing if insurance would cover bariatric surgery.

Conclusion: Decision making by PCPs for severely obese patients seems to underprioritize obesity treatment and overestimate bariatric surgery risks. This could be addressed with PCP education and improvements in communication between PCPs and bariatric surgeons. (Surg Obes Relat Dis 2016;:00-00.) (C) 2016 American Society for Bariatric Surgery. Published by Elsevier Inc. All rights reserved.

The research described in this manuscript was supported by a 2014 ASMBS Research Grant to Dr. Funk. This work was also supported by grant UL1 TR000427 from the Clinical and Translational Science Award (CTSA) program through the National Institutes of Health National Center for Advancing Translational Sciences and by resources from the Department of Veterans Affairs (DVA). The design and conduct of the study, data collection, management, analysis, approval of the manuscript, and decision to submit were made solely by the research team.

*Correspondence: Luke M. Funk, M.D., M.P.H., Assistant Professor of Surgery, University of Wisconsin School of Medicine and Public Health, Department of Surgery, 600 Highland Avenue H4/728 Clinical Science Center, Madison, WI 53792-7375, USA.

E-mail: funk@surgery.wisc.edu 
Although the overall rates of obesity have stabilized in the United States in recent years, the prevalence of severe obesity (body mass index [BMI] of $\geq 35 \mathrm{~kg} / \mathrm{m}^{2}$ ) has increased by $70 \%$, to 18 million, over the past decade [1]. This increase has resulted in significant costs to the US healthcare system. Although they comprise only $37 \%$ of the employed population with obesity (BMI $\geq 30$ ), individuals with a BMI $\geq 35$ generate nearly two thirds of the annual excess costs to employers, attributable to obesity, which amounts to $>\$ 40$ billion per year [2].

Bariatric surgery is the most effective treatment for severe obesity. It achieves substantial and sustained weight loss, co-morbidity resolution, and improvements in quality of life and is associated with extended lifespan [3-5]. A systematic review has indicated that it is cost effective [6,7]. All professional societies that represent physicians who manage severely obese patients-the American College of Cardiology/American Heart Association [8], American Association of Clinical Endocrinologists [9], the Obesity Society [9], the American Society for Metabolic and Bariatric Surgery [9], and the American Academy of Family Physicians (AAFP) [10]—recommend bariatric surgery referral and evaluation for morbidly obese patients considered appropriate surgical candidates. Despite these data, $<1 \%$ of severely obese US adults undergo bariatric surgery annually [11].

Given that a relatively small proportion of eligible patients receive bariatric surgery, it is critical to understand barriers and facilitators to the utilization of bariatric surgery. A recently published systematic review found that both patients and referring practitioners had significant concerns about the outcomes and safety of bariatric surgery, although they admitted they had limited knowledge about obesity treatment options in general [12]. Only 1 study included practitioners as participants and did not assess how the providers made treatment decisions with regard to severe obesity. Rather, it focused on barriers to referral faced by primary care physicians (PCPs) [13]. Another study included in the systematic review found that PCP recommendations were identified as an important predictor of whether a patient would consider bariatric surgery $[12,14]$.

To better understand how PCPs make treatment recommendations for their severely obese patients, we conducted focus groups with PCPs in Wisconsin. We sought to better understand how PCPs prioritize the recommendations made to severely obese patients. We also investigated how PCPs approach bariatric surgery as a treatment option and the challenges they encounter during the referral process.

\section{Materials and methods}

\section{Study design and recruitment}

We conducted 3 focus groups with PCPs who were members of the Wisconsin Research and Education Network (WREN), a statewide practice-based research network of 200 primary care clinicians and $>200$ researchers $[15,16]$. Interested clinicians were asked to complete a short eligibility survey online. Of the 27 clinicians who completed the survey, the 26 who met our eligibility criteria were MDs or DOs, managed adult patients ( $>50 \%$ of their practice), and had evaluated at least 5 severely obese patients (BMI $>35$ or higher) in their clinic over the past 6 months. A member of the University of Wisconsin (UWMadison) Survey Center team called all 26 eligible PCPs and invited them to attend upcoming focus groups scheduled in Mauston (population 4423), Madison (population 233,209), and Milwaukee (population 594,833) [17].

\section{Focus group procedures and guide}

After obtaining written informed consent from participants, a trained moderator facilitated discussion, using a script with prespecified questions and discussion topics (Appendix). The focus group script followed a questioning route [18], which was guided by our study's primary objectives. Participants were given a clinical vignette describing a severely obese (BMI 46) 52-year-old male, who was attending his first PCP visit. His co-morbidities included type 2 diabetes (T2D), hypertension, obstructive sleep apnea, and anxiety. He smoked 1 pack of cigarettes per day. The PCPs were asked how they would prioritize treatment of his multiple health conditions and when, if ever, they would include bariatric surgery as a treatment option. The moderator then asked the participants to discuss their approach to treatment of severe obesity more generally, using open-ended probes to ensure that the key themes were addressed.

The duration of each focus group session was approximately 90 minutes. Upon completion of the session, participants completed an anonymous questionnaire, which included questions on demographics. All sessions were audio-recorded and transcribed. Each focus group participant received $\$ 150$ upon completion of the focus group session.

\section{Qualitative data analysis}

Focus group sessions were analyzed by using a directed approach to content analysis [19]. Three research team 
members (LMF, SAJ, CIV) coded the first transcript independently for emerging themes. Then, they convened to discuss each coded phrase or idea. This procedure was repeated for each subsequent transcript, using the technique of constant comparison and ultimately developing a taxonomy of consensus codes [20]. Memos were created to further clarify code definitions. ATLAS.ti qualitative data analysis software (ATLAS.ti7, Scientific Software Development; Berlin, Germany) was used to manage the data. Codes were exported and divided to create code summaries and identify representative quotes. Code summaries were then aggregated to higher order themes, which were discussed and reviewed by three team members (LMF, SAJ, CIV).

This study was approved by the UW-Madison Education and Social/Behavioral Science Institutional Review Board in March 2014.

\section{Results}

Of the 26 eligible PCPs, 17 reported that they were able to attend focus group sessions, of which 16 attended (3, 7, and 6 in Madison, Mauston, and Milwaukee, respectively). Their average age was 45.7 years $( \pm 11.3 \mathrm{yr}$ ). Of the PCPs, $50 \%$ were female, and $94 \%$ were white.

\section{PCP approaches to prioritizing treatment for severely obese patients}

Approach \#1: Treat the disease that is easiest to address. PCPs prioritized treatment of diseases that they were comfortable managing and that could be treated with specific medications. Treatment of hypertension and T2D first was often justified with this approach. By addressing these conditions, PCPs felt that they were able to establish rapport with patients and have some initial treatment success, which may set the stage for future treatment successes that would require lifestyle changes.

Approach \#2: Treat the disease that is perceived as the most dangerous. Some PCPs reported that they would initially address certain obesity-related co-morbidities, such as T2D or hypertension, because they presented either the most immediate health risk or the greatest health risk. Future development of life-threatening conditions, such as coronary artery disease, heart failure, and renal insufficiency, were concerns for PCPs who used this approach.

Approach \#3: Let the patient set the agenda. Some PCPs let patients decide which co-morbidity they would like to address first, and this empowered patients to take an active role in their health. PCPs reported that if patients were not fully invested in the treatment plan, then it was unlikely to succeed. Patients rarely focused on obesity treatment initially given that the lifestyle and dietary changes needed to achieve sustained weight loss were hard to make.

Approach \#4: Address obesity first because it is the "common denominator" underlying other co-morbid conditions. PCPs who used this approach felt that addressing the patient's obesity would help treat many of the other comorbidities. Without obesity treatment, it would be much more difficult to achieve sustained resolution of other comorbidities (Table 1).

\section{Factors influencing the formation of PCP care plans for obesity}

1. Patient acceptance of the treatment plan. PCPs were cognizant of how patients perceived and accepted a proposed treatment plan. PCPs felt that making strong recommendations for significant weight loss over a short period of time was unlikely to be successful and may harm their relationship with the patient. PCPs often recommended incremental lifestyle changes for obesity treatment, which were positively reinforced during subsequent clinic visits. Behavior change strategies included self-monitoring of dietary habits and physical activity and making incremental dietary changes.

2. Availability of nurse educators, dieticians, and educational materials. Obesity treatment required significant support from additional staff. Nurses who specialized in T2D education and nutritionists who could provide recommendations regarding healthy dietary habits were critical but were not available at all clinics. When present, these staff members also monitored patients between clinic visits, managed medications, and offered education. This helped PCPs overcome the lack of faceto-face time with patients. PCPs also used handouts or other educational materials to compensate for limited face-to-face time.

3. Considering the severity of obesity and co-morbidities. Several PCPs noted that reviewing patient BMI and having it listed prominently in the electronic health record was helpful in care planning. One PCP remarked that he treated BMI like a vital sign. PCPs were less likely to focus on obesity treatment for patients with class I obesity (BMI 30-34.9). Many PCPs became concerned about obesity treatment for patients with class III obesity (BMI $\geq 40$ ) because they felt it was associated with poor long-term health outcomes (Table 2).

\section{Challenges to implementing PCP care plans}

PCPs reported several patient, provider, and health care system challenges to implementing care plans for their obese patients. 
Table 1

Approaches PCPs use to prioritize treatment for severely obese patients and representative quotes

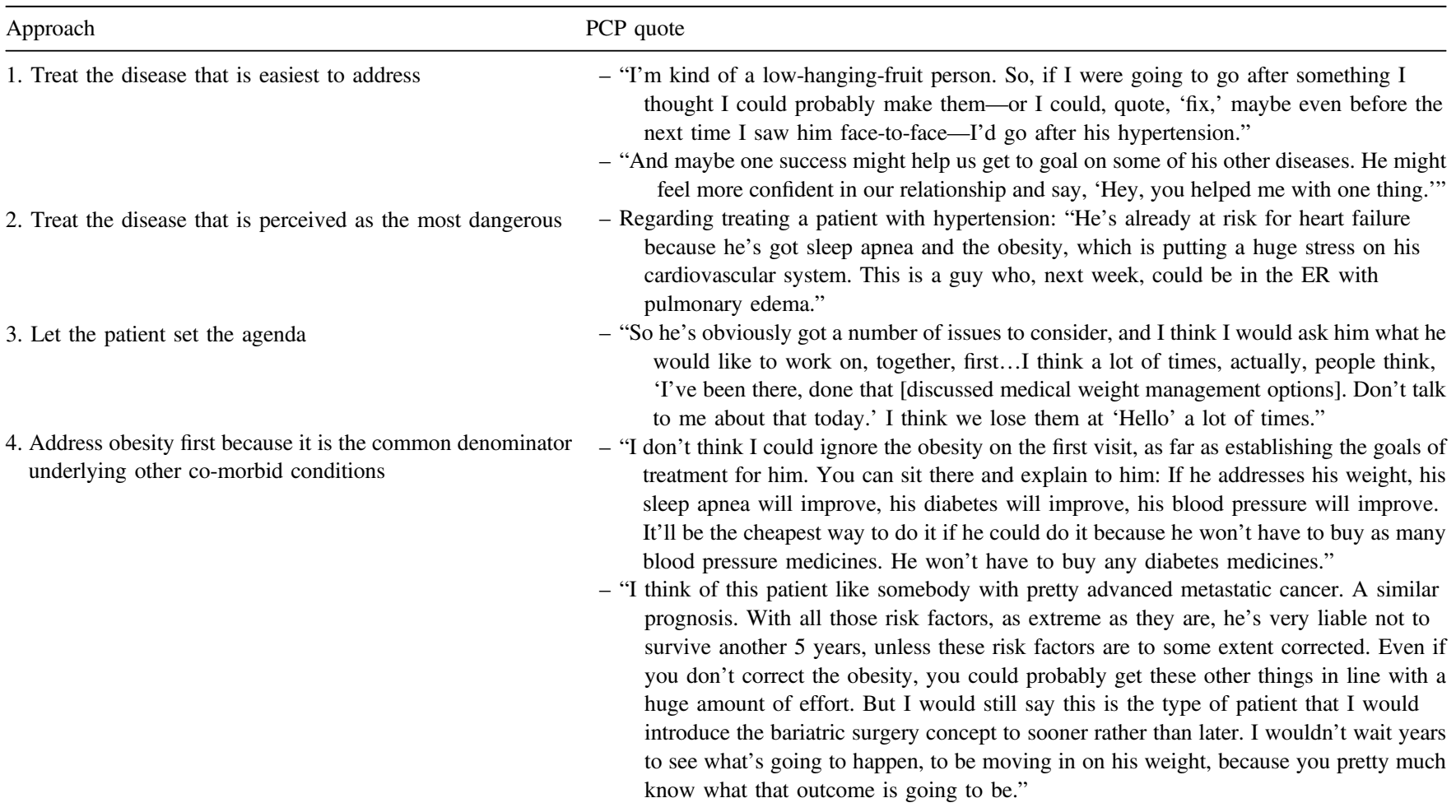

$\mathrm{PCP}=$ primary care physician

\section{Patient factors}

1. Lower socioeconomic status: Severely obese patients often had limited flexibility with regard to their jobs and fewer financial resources, which made it difficult to incorporate healthy eating and physical activity. Lack of family support, such as assistance with child care, limited the time for physical activity.

2. Eating as an addiction: PCPs felt that many obese patients were addicted to calories, and they felt that the physiologic mechanism was similar to that in patients with drug or alcohol addiction in that eating caused "endorphin highs." Making sustained dietary changes required significant changes in patients' lives, including the people with whom they spent time.

3. Having prior weight loss failures: Prior weight loss failures undermined patients' confidence in their ability to be successful with future weight loss attempts and, as such, were barriers to lifestyle change.

4. Being in denial or making excuses: PCPs reported that it was common for patients not to take responsibility for their behavior. Inaccurate reporting of food diaries and statements regarding lack of time, energy, or desire to exercise were common.

5. Limited physical mobility: Although increased physical activity was often recommended by PCPs, severely obese patients had difficulty implementing activity plans because of obesity-related pain.

\section{Practitioner factor.}

1. Feeling ineffective in their ability to help patients lose weight: Lack of patient success frustrated PCPs, which sometimes diminished their enthusiasm to motivate patients to lose weight. PCPs often felt uncomfortable making recommendations for obesity medications, and this contributed to their feelings of ineffectiveness. Their discomfort with making medication recommendations was attributed to their lack of familiarity with obesity medications.

\section{Systems factors.}

1. Poor reimbursement for services: PCPs were often unsure if they were going to be reimbursed for obesityrelated counseling and services because weight loss counseling and referrals to dieticians were often not covered by insurance. When obesity was listed as a primary diagnosis in the progress note, it was harder to receive reimbursement for the care provided (versus T2D or hypertension listed as the primary diagnosis). 
Table 2

Factors influencing the formation and implementation of PCP plans for severely obese patients and representative quotes

Factors influencing formation of care plans

1. Patient acceptance of treatment plan

2. Availability of nurse educators, support staff, and educational materials

- "If you never want to see them again, you know what you say to them? 'You're morbidly obese and you really need to lose 25 pounds before I see you next time'."

- "If I know that we're already short on nursing staff, the last thing I want to do is add to their plate. You need to call this person every week, and you need to do blood pressure checks and weight checks, and I can't physically do that to our staff at the moment."

- "Because, I know I've got a great one-page hand-out, front and back, that says, 'Here's a list of foods, all of which you're familiar with. Pick the list from top to bottom. The bottom ones are always the better choices.' I could easily, probably, spend 2 minutes on that, and it seems to be a fairly highyield sort of improvement for many of these things."

3. Considering the severity of obesity and comorbidities

- "There are people with BMIs of 32 that don't have arthritis and diabetes, and they're active, and they're firefighters and, you know, maybe it's genetics or build or whatever... I have a real hard time [critiquing] them because they're over insurance companies' standards for weight. They're maybe the healthy overweight, per se, or they're big muscular builds or whatever. So, that's why I let some people get away without as much lecturing as others. But usually, I guess my line seems to be a BMI of 40."

- "If I haven't looked at a BMI at the beginning of an encounter and then catching it at the end, and being like, 'Oh, we should've talked about that and that wasn't even at the top of my [list].' I wouldn't have known if I hadn't seen the [BMI], and I think that's my own normalization [because] so many of our patients are obese."

Challenges to implementing care plans

1 A. Lower socioeconomic status (patient level)

- "That single parent, sometimes they're just, they're strapped, they're exhausted, they've worked all day, they go back to their 3 kids. It's faster for them to hit the dollar menu at McDonald's than to prepare a healthy meal. And she can feed herself and her 3 kids cheaper off the dollar menu than she really can prepare a healthy meal for $4 . "$

1 B. Eating as an addiction (patient level)

- "If certain types of foods were illegal, would it be all that much different from illicit substances? Probably not. A lot of people are addicted to certain types of calories."

- "When I talk to patients, I liken it to other addictions, like tobacco or drugs. You know, except it's calories. You have to change who you hang out with, where you go..."

1 C. Having prior weight loss failures (patient level)

1 D. Being in denial/making excuses (patient level)

1 E. Limited physical mobility (patient level)

- "Most of the morbidly obese patients we see have, at least once in their adult life, tried to lose weight or tried to manage their weight. Prior failures, I think, for a lot of my morbidly obese patients,... is a big barrier to them feeling like they could be successful if they embarked again on an attempt to lose weight."

- "If you talk to him, he swears he's under 2000 calories a day. You talk to his wife, and she says he's eating bags of potato chips and boxes of food and, you know, a whole chicken at a time."

- "It's hard to exercise when your [BMI is] 46. You're just creaky. It's hard to get moving and probably takes a lot of energy expended to actually move. But I find with these morbidly obese patients, it's very hard to find some kind of aerobic exercise that they can actually do."

2. Feeling ineffective in helping patients lose weight (practitioner level)

- "I have a panel of 1500 patients at this point. I can think of one that has successfully lost 50, 60 pounds. It was similar to this patient. But that's one out of probably a thousand morbidly obese patients."

- "If it is a case like his wife made him come in, he's probably not going to be too interested in making a whole lot of changes in all these things. And that impacts how much I'm going to bang my head against the wall, as well as what I'm going to kind of expect."

3 A. Poor reimbursement for services (system level)

3 B. Culture promoting obesity (system level)

- "I've had some roadblocks, where people are obese or morbidly obese and I've tried to refer them for dietary counseling, and [we were] denied because whatever insurance they had, they could get dietary counseling for diabetes only, not for obesity."

- "In America, we don't eat because we're hungry. We eat for comfort. We eat for pleasure. We eat for socializing."

$\mathrm{BMI}=$ body mass index $; \mathrm{PCP}=$ primary care physician

2. Culture promoting obesity: PCPs believed that American culture contributed to the obesity epidemic by promoting eating for comfort or pleasure. PCPs felt that the food industry played a large role in encouraging unhealthy food choices (see Table 2).

\section{Factors influencing bariatric surgery referral}

PCPs rarely, if ever, brought up the option of bariatric surgery with patients. One PCP remarked, "I don't bring it up unless people talk to me about it first because I think I 
need to have that kind of interface before I can even have a discussion about risk. I consider bariatric surgery to be risky." Waiting for patients to broach bariatric surgery originated from 5 factors:

1. Wanting to "do no harm." PCPs were concerned about the safety of bariatric surgery and the risk of complications, including poor quality of life, reoperations, and mortality. They noted a mismatch between the published literature, which characterizes bariatric surgery as very safe, and their professional experiences and observations. For patients with BMIs $>40$ but no known obesityrelated co-morbidities, PCPs felt that not recommending bariatric surgery was consistent with "doing no harm." Despite these concerns, there was general consensus that untreated class III obesity was dangerous.

2. Questioning long-term effectiveness of bariatric surgery. Most PCPs believed bariatric surgery was effective in the short-term and cited improvements in quality of life and co-morbidity resolution. However, they expressed concern that long-term failures were common, particularly weight regain or excessive weight loss.

3. Having limited knowledge about bariatric surgery. PCPs generally did not feel confident in their knowledge of bariatric surgery. Most were not sure, for instance, if it was routinely performed via the open approach or the laparoscopic approach. There was limited familiarity with laparoscopic vertical sleeve gastrectomy, which is the most commonly performed bariatric operation in the the United States.

4. Not wanting to recommend bariatric surgery too early. PCPs wanted to recommend dietary (and sometimes medication) changes first to ensure that patients were "engaged" before recommending bariatric surgery. PCPs felt that patients who were motivated had the best outcomes, whereas those who were looking for a "quick fix" or primarily cosmetic benefit were less likely to succeed.

5. Not knowing if insurance will cover bariatric surgery. PCPs stated that insurance was a major barrier for bariatric surgery referral. PCPs did not want to refer their patients for bariatric surgery if the request would ultimately be rejected by the insurance company (Table 3).

\section{Challenges to pursuing bariatric surgery}

Patient factors.

1. Meeting preoperative requirements: PCPs noted that bariatric programs had rigorous preoperative requirements, including assessments from a health psychologist, nutritionist, bariatric surgeon, and occasionally medical subspecialists. Many insurers required several months of supervised medical weight loss attempts, and bariatric programs often had a weight loss requirement before surgery. Dietary changes and other lifestyle changes, such as smoking cessation and improvements in blood glucose control for patients with T2D, were frequently required. Although viewed by PCPs as reasonable, these requirements were difficult to meet for some patients.

2. Living far from a bariatric surgery program: Living far from a bariatric surgery program presented logistical difficulties because of the multiple visits to the bariatric clinic and hospital required to complete their preoperative evaluations, surgery, and postoperative care.

Practitioner factor. PCP involvement in postoperative care: Although many bariatric programs counseled patients to follow-up with the bariatric program annually for the rest of their lives, PCPs were often involved in postoperative care. This may include laboratory checks on vitamin levels, wound monitoring, and evaluation of abdominal pain. The requirement of PCPs to address these issues contributed to the hesitation to refer patients for bariatric surgery (Table 3).

\section{Discussion}

Our PCP participants utilized several different approaches when prioritizing care for severely obese patients. Three of the 4 prioritization approaches placed the emphasis on obesity-related co-morbidities, but not on obesity itself. Obesity was rarely seen as the "most dangerous" disease. Hypertension and T2D were often perceived as "easier" to treat because effective medications could be prescribed [21]. Patients were often not interested in addressing their obesity and would usually not "set the agenda" to prioritize obesity treatment.

If the PCP and the patient decided to treat the patient's obesity, there were numerous challenges from the PCP's perspective, including low socioeconomic status, prior weight loss failures, and limited reimbursement. These could not be effectively addressed by PCPs in the clinic, so they frequently felt inadequate with regard to helping their patients lose weight. This finding is consistent with a survey by Ferrante, who found that most PCPs felt that treating obesity was frustrating and often ineffective [13].

Many of the concerns expressed by PCPs about bariatric surgery can be addressed through education and improved communication with bariatric surgeons and patients. Likewise, many of the barriers to bariatric surgery referral cited by our participants, including when patients should be referred for bariatric surgery, bariatric program approval criteria, and PCP involvement in postoperative care, can be mitigated by improved communication. Numerous studies have demonstrated that bariatric surgery is safe and 
Table 3

Factors influencing bariatric surgery referral and representative quotes

Factors influencing whether PCPs provide bariatric surgery referrals

1. Wanting to "do no harm"

2. Questioning long-term effectiveness of bariatric surgery

3. Having limited knowledge about bariatric surgery

4. Not wanting to recommend bariatric surgery too early

5. Not knowing if insurance will cover bariatric surgery
- "There are some times when I think, 'Boy, I'd really like to have this patient have [bariatric surgery],' but I am a little afraid that with all these other co-morbidities, their risk of complications [is] very high."

- "You read these articles right out of the New England Journal of Medicine: incredibly well-tolerated, and then you see patients who are disasters, and you're trying to put it altogether. Are we missing something here? Are they selecting their patients so carefully that when you're played over broader population like we see you can get disasters that you didn't see in the study?"

- "I've had another patient...she lost a lot of weight with surgery about 20 years ago. Gained it all back."

- "I'd say about $50 \%$ of the patients I know who had [bariatric surgery] have either outeaten it or gained back most of the weight, and they are well on their way to the same complications they had before surgery."

- "So, I actually have a patient, where she is so skinny [after bariatric surgery], at this point and can't absorb any nutrients, that we admit her to our inpatient service every month. So I think it goes both ways."

- Regarding whether bariatric surgery is typically performed laparoscopically or open: "I think most of the [bariatric operations] I've seen have been open, but, to be honest, I don't truly know that because I think people had to have revisions and multiple abdominal surgeries afterward. So, I don't truly know what the initial one would be."

- "Often I feel I want to get their medical problems like diabetes and blood pressure well-controlled before I make a referral because, certainly if they have an $\mathrm{HbA} 1 \mathrm{C}$ of 10 or blood pressures of 160 s over 80 s, they're not in the best control possible. They're not going to be a good surgical candidate anyway. So again, if the patient would have asked [for a bariatric surgery referral] at his first visit, I'd say 'No. [let's] fix these things well before we consider that. You need to try and fail multiple options that could be effective for you. And remembering again, bariatric surgery may help you lose weight but you still have medical problems we need to address. Those still remain lifestyle changes, and medications need to be a part of that."

- "I can't keep [the insurance] straight, so, therefore, I don't know even if it's covered. Under what circumstances is it covered? At what BMI threshold is it covered, even if it is covered? Therefore, I'm naive to, can I even offer this to him or should I not set him up for anticipation and excitement about possible treatment, that he then crashes and burns and loses all faith in me because I said something was going to be available, an opportunity for him that truly didn't exist?"

- "I never bring [bariatric surgery] up because, usually, I assume it's not covered, and for a lot of my patients, it's not financially an option for that out-of-pocket at all."

Challenges to pursuing bariatric surgery

1 A. Meeting preoperative requirements (patient)

- "He sees [the bariatric surgery team] every 2 weeks or at least every 4 weeks. He's been doing this for a year. He's getting very disappointed because they are telling him, 'You have to make big lifestyle changes, dietary changes,' and, of course, if he was successful, he wouldn't need the bariatric surgery. But even with a year of intensive therapy, more than I can give my usual patient, his BMI remains 77. This is one year of a major bariatric center, throwing the kitchen sink at him, to try to prepare him for upcoming bariatric surgery, which they're promising him. He's getting very frustrated, because they have not cleared him for surgery yet, and he's been in this process for a year, and he's also not lost weight."

1 B. Living far from a bariatric surgery program (patient)

2. Primary care practitioner involvement in postoperative care (practitioner)
- "I'm in XXX County, and, as far as I know, there's not a bariatric program there. We generally refer to XXX County, to their bariatric program. So, that is a barrier of location. Many of my patients in XXX think Milwaukee is like Chicago. It's 20 minutes away, but, to them, that's very far out to go to have to go to Milwaukee and see physicians."

- "And again, ignorance here, I don't quite know exactly what the frequency of what should be tested and what exactly should be tested and if it's my responsibility as the primary doctor to manage those potentially malabsorption issues. Or should it be, in my opinion, maybe the surgical team and the nutritionist associated with that team during the follow-up and verifying that all the T's and I's are dotted and crossed."

$\mathrm{BMI}=$ body mass index $\mathrm{PCP}=$ primary care physician

effective $[3,4,22,23]$. There is also strong observational data suggesting that bariatric surgery is beneficial for at least 10 to 20 years [5,24]. Increased dissemination of this evidence to PCPs and medical trainees may lead to prioritization of obesity treatment. Furthermore, discussions about medical and surgical weight management options are warranted early in the course of the patient's disease. Such discussions not only inform patients about the health risks of ongoing 
obesity but also provide knowledge about risks, benefits, and outcomes of treatments. Decision aids that facilitate shared decision making could help providers optimize care for individuals with severe obesity.

Other PCP concerns, such as the long-term effectiveness of bariatric surgery, cannot be addressed solely with education and improved communication. Although bariatric surgery has been shown to provide a mortality benefit as far as 10 years following surgery [5], there are notable gaps in the literature. Weight loss and co-morbidity resolution outcomes $>10$ years after bariatric surgery are poorly reported. Weight regain is a legitimate concern, with one study reporting that $37 \%$ of patients regained at least $25 \%$ of their total lost weight at a mean of 7 years after gastric bypass [25]. To address these issues, more long-term data are needed, and the clinical approach to postoperative weight regain should be closely examined.

Furthermore, numerous professional societies, such as the AAFP, do not endorse or explicitly support bariatric surgery. In their document "Diagnosis and Management of Obesity," published in 2013, the AAFP noted that bariatric surgery "may be considered in adults who have not achieved weight loss with dietary or other treatments" [10]. Given the relatively neutral position taken by the AAFP, it is not surprising that PCPs do not strongly endorse bariatric surgery. However, the extent of PCPs' awareness of the AAFP's position on bariatric surgery and the way it impacts their treatment recommendations are still unclear.

Establishment of standardized obesity management metrics would also be helpful because they would improve our understanding of how severely obese patients are being managed at the healthcare system, state, and national levels. Analogous to those developed for surveillance of surgical quality and safety on a global level, these metrics should be based on simplicity, wide applicability, relevance to public health, and minimizing negative consequences of measurement [26]. They could incorporate various structure (i.e., number of practitioners), process (i.e., number of patients with a record of their BMI; number referred to nutritionist or bariatric program) and outcome (i.e., number who underwent bariatric surgery; BMI changes over time) measures to assess the quality of medical care provided to a population [27]. Although some groups, such as the National Committee for Quality Assurance, have incorporated BMI measurement and such components into their quality measures [28], a more comprehensive set of measures, including obesity treatment outcomes that are readily available to providers, researchers, and policymakers, seems warranted.

Our study has several limitations. Given that our approach was qualitative rather than quantitative, we cannot infer the prevalence of PCP attitudes about severe obesity care. Our results were also limited to areas of discussion that were generated from our interview guide. We attempted to minimize the likelihood of missing important topics by combining our clinical vignettes with open-ended questions for the providers. Furthermore, although we selected PCPs from different practice locations and included equal numbers of men and women, $>90 \%$ of our PCPs were white. Thus, our findings may not be generalizable to physicians with other characteristics or with different patient populations. Finally, given that obesity care occurs over multiple visits, PCPs may have identified with more than one prioritization approach. We did not attempt to quantify how many approaches were endorsed by each PCP.

\section{Conclusion}

The current practice environment makes it difficult and frustrating for PCPs to medically manage severe obesity successfully. Although PCPs believe that bariatric surgery is effective, they have concerns about its long-term benefits. Severe obesity care can be substantially improved with improvements in communication, PCP and patient education, establishment of standardized metrics, and additional research. These are potentially high-impact areas from a public health perspective and should be prioritized.

\section{Disclosures}

The authors report no proprietary or commercial interest in any product mentioned or concept discussed in this article.

\section{Acknowledgments}

We would like to thank Dr. Kenneth Croes (Senior Project Director, UW-Madison Survey Center), who assisted with focus group recruitment and moderated each focus group. We would also like to thank Dr. David Hahn, M.D., M.S. (Director of the Wisconsin Research and Education Network), who assisted with recruitment of primary care physicians through the Wisconsin Research and Education Network.

\section{Appendix}

\section{Supplementary data}

Supplementary data associated with this article can be found in the online version at http://dx.doi.org/10.1016/j. soard.2015.11.028.

\section{References}

[1] Sturm R, Hattori A. Morbid obesity rates continue to rise rapidly in the United States. Int J Obes (Lond) 2013;37(6):889-91.

[2] Finkelstein EA, DiBonaventura M, Burgess SM, Hale BC. The costs of obesity in the workplace. J Occup Environ Med 2010;52 (10):971-6.

[3] Chang SH, Stoll CR, Song J, et al. The effectiveness and risks of bariatric surgery: an updated systematic review and meta-analysis, 2003-2012. JAMA Surg 2013;149(3):275-87. 
[4] Schauer PR, Bhatt DL, Kirwan JP, et al. Bariatric surgery versus intensive medical therapy for diabetes-3-year outcomes. N Engl J Med 2014;370(21):2002-13.

[5] Arterburn DE, Olsen MK, Smith VA, et al. Association between bariatric surgery and long-term survival. JAMA 2015;313 (1):62-70.

[6] Wang BC, Furnback W. Modelling the long-term outcomes of bariatric surgery: a review of cost-effectiveness studies. Best Pract Res Clin Gastroenterol 2013;27(6):987-95.

[7] Finkelstein EA, Trogdon JG, Cohen JW, Dietz W. Annual medical spending attributable to obesity: payer-and service-specific estimates. Health Aff (Millwood) 2009;28(5):w822-31.

[8] Executive summary: Guidelines (2013) for the management of overweight and obesity in adults: a report of the American College of Cardiology/American Heart Association Task Force on Practice Guidelines and the Obesity Society published by the Obesity Society and American College of Cardiology/American Heart Association Task Force on Practice Guidelines. Based on a systematic review from the The Obesity Expert Panel, 2013. Obesity (Silver Spring) 2014;22(Suppl 2):S5-39.

[9] Mechanick JI, Youdim A, Jones DB, et al. Clinical practice guidelines for the perioperative nutritional, metabolic, and nonsurgical support of the bariatric surgery patient-2013 update: cosponsored by American Association of Clinical Endocrinologists, the Obesity Society, and American Society for Metabolic \& Bariatric Surgery. Endocr Pract 2013;19(2):337-72.

[10] Diagnosis and Management of Obesity [mongraph on the Internet]. Leawood, KS: AAFP; 2013 [cited 2015 Mar 26]. Available from: http://www.aafp.org/dam/AAFP/documents/patient_care/fitness/obesi ty-diagnosis-management.pdf.

[11] Nguyen NT, Masoomi H, Magno CP, et al. Trends in use of bariatric surgery, 2003-2008. J Am Coll Surg 2011;213(2):261-6.

[12] Funk LM, Jolles S, Fischer LE, Voils CI. Patient and referring practitioner characteristics associated with the likelihood of undergoing bariatric surgery: a systematic review. JAMA Surg 2015;150 (10):999-1005.

[13] Ferrante JM, Piasecki AK, Ohman-Strickland PA, Crabtree BF. Family physicians' practices and attitudes regarding care of extremely obese patients. Obesity (Silver Spring) 2009;17(9):1710-6.
[14] Wee CC, Davis RB, Chiodi S, Huskey KW, Hamel MB. Sex, race, and the adverse effects of social stigma vs. other quality of life factors among primary care patients with moderate to severe obesity. J Gen Intern Med 2015;30(2):229-35.

[15] Wisconsin Research \& Education Network: About WREN [homepage on the Internet]. Madison, WI: WREN; 2015 [cited 2015 Jun 12]. Available from: http://www.fammed.wisc.edu/research/wren/about.

[16] Proceedings from the 2008 Wisconsin Quality and Safety Forum, part I. WMJ 2008;107(8):382-8.

[17] United States Census Bureau: American FactFinder [cited 2015 Jun 9]. Available from: http://factfinder.census.gov/faces/tableservices/jsf/ pages/productview.xhtml?src=bkmk.

[18] Krueger R. A practical guide for applied research. Thousand Oaks, CA: Sage Publications; 1994.

[19] Hsieh HF, Shannon SE. Three approaches to qualitative content analysis. Qual Health Res 2005;15(9):1277-88.

[20] Charmaz K. Constructing grounded theory: a practical guide through qualitative analysis. London, UK: Sage Publications, Ltd; 2006.

[21] Colbert JA, Jangi S. Training physicians to manage obesity—back to the drawing board. N Engl J Med 2013;369(15):1389-91.

[22] Mingrone G, Panunzi S, De Gaetano A, et al. Bariatric surgery versus conventional medical therapy for type 2 diabetes. N Engl J Med 2012;366(17):1577-85.

[23] Ikramuddin S, Korner J, Lee WJ, et al. Roux-en-Y gastric bypass vs intensive medical management for the control of type 2 diabetes, hypertension, and hyperlipidemia: the Diabetes Surgery Study randomized clinical trial. JAMA 2013;309(21):2240-9.

[24] Neovius M, Narbro K, Keating C, et al. Health care use during 20 years following bariatric surgery. JAMA 2012;308(11):1132-41.

[25] Cooper TC, Simmons EB, Webb K, Burns JL, Kushner RF. Trends in weight regain following Roux-en-Y gastric bypass (RYGB) bariatric surgery. Obes Surg 2015;25(8):1474-81.

[26] Weiser TG, Makary MA, Haynes AB, et al. Standardised metrics for global surgical surveillance. Lancet 2009;374(9695):1113-7.

[27] Donabedian A. Evaluating the quality of medical care. Milbank Mem Fund Q 1966;44(3):166-206.

[28] Arterburn DE, Alexander GL, Calvi J, et al. Body mass index measurement and obesity prevalence in ten U.S. health plans. Clin Med Res 2010;8(3-4):126-30. 